\title{
Some dynamical properties of S-unimodal maps
}

\author{
by
}

\author{
Tomasz Nowicki (Warszawa)
}

\begin{abstract}
We study 1) the slopes of central branches of iterates of S-unimodal maps, comparing them to the derivatives on the critical trajectory, 2) the hyperbolic structure of Collet-Eckmann maps estimating the exponents, and under a summability condition 3) the images of the density one under the iterates of the Perron-Frobenius operator, 4) the density of the absolutely continuous invariant measure.
\end{abstract}

1. Introduction. The study of S-unimodal maps of the interval (definitions may be found in the next section, for general reference see [CE1, $\mathrm{MS}]$ ) as examples of simple dynamical systems with complicated dynamics is already quite developed during the last ten years. Conditions are known under which the system exhibits different types of asymptotical behaviour. It is generally admitted that the crucial role in this system is played by the critical point, its neighbourhoods and its trajectory. The counterplay between the contraction near the critical point and the expansion along the further critical trajectory may be observed in the derivatives of the iterates at the critical value.

Two conditions on these derivatives seem to be worth studying, both securing the existence of an absolutely continuous invariant measure (acim for short). Such a measure is ergodic [BL], and has a density separated from zero on its support (see $[\mathrm{K}]$ ). It follows that it has singularities of at least root type along the critical trajectory. It is interesting to see that under some relatively weak assumption on the derivatives of the critical trajectory one can estimate the density of the acim explicitly from above. This estimate is sharp in the sense that it only allows those singularities (of the type and in the place) which actually have to appear.

THEOREM A. Suppose that a nonflat S-unimodal function $f$ satisfies the summability condition on the critical trajectory, i.e.

Supported by an Alexander von Humboldt Grant. 


$$
\sum_{r=0}^{\infty} D f^{r}\left(c_{1}\right)^{-1 / l}<\infty .
$$

Then $f$ has an acim $\mu$ with density $h$ such that

$$
h(x)<\mathrm{const} \sum_{r=1}^{\infty} D f^{r-1}\left(c_{1}\right)^{-1 / l}\left|x-c_{r}\right|^{-(1-1 / l)} .
$$

(Here $c_{n}=f^{n}(c)$, where $c$ - the unique critical point of $f$-is not flat, i.e. $\left|f^{\prime}(x)\right|=\mathcal{O}(1)|x-c|^{l-1}, l>1$, and $\left.D f^{n}=\left|\left(f^{n}\right)^{\prime}\right|.\right)$

This condition, the proof of the existence of $\mu$ and the property that $\mu(A)<$ const $|A|^{-1 / l}$ first appeared in [NS3]. Here we give an independent proof which yields the estimate of $h$ (see Corollary 4.2 and the following remarks).

The existence of an ergodic acim $\mu$ implies [L] a hyperbolic structure on the interval in the following sense: for almost all points $x$ of the interval the limit $\lambda_{x}:=\lim _{n \rightarrow \infty} D f^{n}(x)^{1 / n}$ exists, equals $\lambda_{f}$, and $\log \lambda_{f}>0$ is the metric entropy of $f$ with respect to $\mu$. On the other hand, by $[\mathrm{K}]$ the existence of some hyperbolic structure (i.e. $\bar{\lambda}_{x}=\lim \sup D f^{n}(x)^{1 / n}>1$ on a set of positive Lebesgue measure) implies the existence of $\mu$. The problem is therefore to study the uniformity of this hyperbolic structure. Because of the existence of critical points for the iterates one can only consider this uniformity on some special sets [N1-N3, NS1].

Theorem B. For a Collet-Eckmann map $f$, i.e. when

$$
\liminf D f^{n}\left(c_{1}\right)^{1 / n}=\lambda_{C}>1,
$$

the uniform hyperbolic structure is bounded from below by $\lambda_{C}^{1 / \ell}$. In particular, $\lambda_{f} \geq \lambda_{C}^{1 / \ell}$.

For a more precise formulation of this result and the proof see Proposition 3.2. Such maps were introduced in [CE2] where it was shown that they have an acim. From this point of view some generalization was studied in [NS2], where the S-condition was skipped.

The main tools in the present paper are delicate estimates of the mean slope of some special (central) intervals of monotonicity of the iterates of $f$ by the derivatives on the critical trajectory. This is described in Proposition 3.1. The estimates of the density follow from a careful study of the derivatives (Lemma 4.1) and the uniform (in iterates) summability of the inverses of the mean slopes on some crucial intervals of monotonicity of iterates of $f$.

\section{Remarks on Theorem A}

Remark 1.1. It is still an open question if the acim $\mu$ of an S-unimodal map may have thicker singularities. If $\mu(A)<$ const $|A|^{1 / l}$ for any measur- 
able $A$ then by carefully choosing small sets near the critical trajectory and using Keller's result that $\mu(A)>$ const $|A|$ one obtains $\sum D f^{n}\left(c_{1}\right)^{-1 /(l-1)}$ $<\infty$.

Remark 1.2. The same bounds for the density were found by Szewc [Sz] for Misiurewicz maps [M]. Recently similar bounds (with $(1.9)^{-r}$ instead of $\left.D f^{r}\left(c_{1}\right)^{-1 / l}\right)$ were found by L.-S. Young [Y] for a subclass of ColletEckmann maps. For Collet-Eckmann maps it was proven in $[\mathrm{KN}]$ that the density is of the form

$$
\phi(x) \sum_{r=1}^{\infty} \lambda^{-r}\left|x-c_{r}\right|^{-(1-1 / l)},
$$

where $\phi$ is of bounded variation and $\lambda$ is defined by the uniform hyperbolic structure of the map (compare Theorem B and Proposition 3.2).

2. Definitions. We shall deal with a nonflat S-unimodal function $f$ of the interval $[0,1]$ into itself. The nonflatness means that there is a constant $M>0$ such that $M<\left|f^{\prime}(x)\right| /|x-c|^{l-1}<M^{-1}$ for any $x \in[0,1]$ and the unique critical point $c(l>1)$. The consequences of nonflatness are stated in Lemma 2.2. We assume that $S f<0$ where $S f=f^{\prime \prime \prime} / f^{\prime}-\frac{3}{2}\left(f^{\prime \prime} / f^{\prime}\right)^{2}$. It follows that $S f^{n}<0$ where $f^{n}$ denotes the $n$th iterate of $f$; moreover (denoting by $|I|$ the length of the interval $I$ ), we have

LEMMA 2.1 (Consequences of $S f<0$ ). Let $g=f^{n}$ and assume that $g_{\mid(a, b)}^{\prime} \neq 0$ and $(x, y) \subset(a, b)$. Then

(1) (minimum principle) $\left|g^{\prime}\right|$ has no minimum in $(a, b)$,

(2) (slope comparing)

$$
\frac{|g(x, y)|}{|(x, y)|} \geq \min \left\{\frac{|g(a, x)|}{|(a, x)|}, \frac{|g(a, y)|}{|(a, y)|}, \frac{|g(y, b)|}{|(y, b)|}\right\},
$$

(3) (Koebe Lemma) for any $\tau>0$ there is a constant $\mathcal{C}(\tau)(\mathcal{C}$ independent of $n$ and $\mathcal{C} \rightarrow 1$ as $\tau \rightarrow \infty)$ such that if $|g(x, a)|>\tau|g(x, b)|$ then

$$
\left|g^{\prime}(x)\right|>\mathcal{C}(\tau)\left|g^{\prime}(b)\right|
$$

(4) (extending cross-ratio)

$$
\frac{|g(a, b)|}{|(a, b)|} \frac{|g(x, y)|}{|(x, y)|}>\frac{|g(a, x)|}{|(a, x)|} \frac{|g(y, b)|}{|(y, b)|},
$$

and in particular when $x=y$

$$
\left|g^{\prime}(x)\right|>\frac{|(a, b)|}{|g(a, b)|} \frac{|g(a, x)|}{|(a, x)|} \frac{|g(x, b)|}{|(x, b)|},
$$


(5) if $a<x<y<b, g^{\prime}(a) \geq 0$ and $g^{\prime \prime}(b)=0$ then

$$
\frac{g^{\prime \prime}(y)}{g^{\prime}(y)^{3 / 2}}<2 \frac{\sqrt{g^{\prime}(y)}-\sqrt{g^{\prime}(x)}}{g(y)-g(x)}<\frac{g^{\prime \prime}(x)}{g^{\prime}(x)^{3 / 2}} .
$$

Proof may be found in [CE1, MS]. The proof of (5) follows by two integrations of $S g<0$.

Lemma 2.2 (Consequences of the nonflatness). Assume that $f$ is nonflat. Then there exists a constant $m>0$ such that

(1) $m<|f(x, c)| /|(x, c)|^{l}<m^{-1}$,

(2) for any $y, x$ with $y \in(x, c)$

$$
\frac{|f(x, c)|}{|(x, c)|} \geq m \frac{|f(x, y)|}{|(x, y)|},
$$

(3) there is a constant $\kappa<1$ such that if $(c, y) \subset(c, x)$ and $|(y, x)|<$ $\kappa|(c, y)|$ then $|f(y, x)|<|f(c, y)|$ and

$$
\kappa \frac{|f(y, x)|}{|(y, x)|}<\frac{|f(y, c)|}{|(y, c)|} .
$$

Proof. (2) follows by comparing both sides with $f^{\prime}(x)$. As to (3) it is enough to observe that by nonflatness we have $|f(y, x)|<|f(c, y)|$ const $\times$ $\kappa(1+\kappa)^{l-1}$ and by (1), (2)

$$
\kappa \frac{|f(y, x)|}{|(y, x)|}<\kappa \frac{(1+\kappa)^{\ell-1}}{m^{3}} \frac{|f(y, c)|}{|(y, c)|} .
$$

By $\Delta_{n}=\left\{\Delta_{n}^{i}\right\}$ we denote the family of all maximal intervals of monotonicity of $f^{n}$, and $\Delta_{n}(x) \in \Delta_{n}$ is the interval containing $x$. We are mostly interested in the intervals from $\widetilde{\Delta}_{n}=\Delta_{n+1} \backslash \Delta_{n}$, and especially in $\widetilde{\Delta}_{n}(c)$, which, when nonempty for some $n$, contains two intervals $(\alpha, c)$ and $(c, \widehat{\alpha})$, with $\alpha \neq \widehat{\alpha}$ and $f(\alpha)=f(\widehat{\alpha})$. There is no essential dynamical difference between these intervals and we shall denote them also by $\widetilde{\Delta}_{n}(c)$.

The images of the intervals from $\widetilde{\Delta}_{n}$ under $f^{n}$ always contain the critical point $c$ as an endpoint - we say that they are at the $c$-level. In particular, $f^{n} \widetilde{\Delta}_{n}(c)=\left(c_{n}, c\right)$.

There is some relation between $\Delta_{n}$ and $\widetilde{\Delta}_{k}$. Namely, for any $I \in \Delta_{n}$ there is a $k<n$ such that $I \in \widetilde{\Delta}_{k}$ and $f^{n} I=f^{n-k} \Delta_{n-k}(c)$. For any $J \in \widetilde{\Delta}_{n}^{n}$ one has a $k<n$ such that $f^{k} J=\widetilde{\Delta}_{n-k}(c)$.

Let $\widetilde{\Delta}_{n}(c) \subset \Delta_{n}(c)=\widetilde{\Delta}_{k}(c)$. Then

$$
\begin{gathered}
f^{k}\left(\widetilde{\Delta}_{k}(c) \backslash \widetilde{\Delta}_{n}(c)\right)=\widetilde{\Delta}_{n-k}(c) \subset \Delta_{n-k}(c), \\
f^{n}\left(\Delta_{n}(c) \backslash \widetilde{\Delta}_{n}(c)\right)=f^{n-k} \widetilde{\Delta}_{n-k}(c)
\end{gathered}
$$


and

$$
f^{n} \widetilde{\Delta}_{n}(c) \subset f^{n-k}\left(\Delta_{n-k}(c) \backslash \widetilde{\Delta}_{n-k}(c)\right) .
$$

In $[\mathrm{N} 2, \mathrm{~N} 3]$ the (two) intervals $\widetilde{\Delta}_{n}(c)$ were called $*(n)$ and the intervals from $\widetilde{\Delta}_{n}$ were called $* *(n)$. We shall use the notation $\sum^{*}$ to indicate summation over all $n$ for which a $\widetilde{\Delta}_{n}(c)$ exists, and $\sum^{* *(n)}$ for sums over all intervals $\widetilde{\Delta}_{n}^{i}$ from $\widetilde{\Delta}_{n}$ for a fixed $n$.

By $D f^{n}$ we understand the absolute value $\left|\left(f^{n}\right)^{\prime}\right|$, and by $\mathcal{O}$ various constants independent of iteration and points. We denote $f^{k}(x)$ by $x_{k}$, in particular $c_{1}=f(c)$ is the critical value of $f$.

3. Central slopes. In this section we estimate the central slopes, i.e. $\left|f^{n} \widetilde{\Delta}_{n}(c)\right| /\left|\widetilde{\Delta}_{n}(c)\right|$, with the aid of the derivatives at the critical value (Proposition 3.1). Together with Corollary 4.1 this gives an independent proof of the existence of an absolutely continuous invariant measure in case of summability on the critical trajectory, and estimates its density. Moreover, we obtain good estimates on different uniform hyperbolic structures in the case of Collet-Eckmann maps.

LEMMA 3.1. Let $A=(c, y)=\widetilde{\Delta}_{m}(c) \in \Delta_{m+1} \backslash \Delta_{m}$. If $\left|f^{m} A\right| /|f A|>$ $\eta D f^{m-1}\left(c_{1}\right)>0$ then for some constant $K_{1}$ due uniquely to the nonflatness of $f$

$$
\left|f^{m} A\right| /|A|>K_{1}\left(\eta D f^{m}\left(c_{1}\right)\right)^{1 / l} .
$$

Proof follows from $|f A|<\mathcal{O}|A|^{l}$ and

$$
\left|f^{m} A\right|^{l-1}=\left|c_{m}-c\right|^{l-1}>\mathcal{O D} f\left(c_{m}\right) \text {. }
$$

LEMmA 3.2. Let $(c, y) \subset(c, x)=\widetilde{\Delta}_{k}(c)=\Delta_{m}(c)$ and $A=(c, y), B=$ $(y, x) \in \widetilde{\Delta}_{m} \quad\left(\right.$ then $\left.f^{k} B=\widetilde{\Delta}_{m-k}(c)\right)$.

(1) Suppose that either $\left|f^{m} A\right|<\tau\left|f^{m} B\right|$ for some $\tau>1$, or $\left|f^{m} A\right| /|A|<$ $\kappa\left|f^{m} B\right| /|B|$ for $\kappa$ from Lemma $2(3)$. Then there exist a constant $\mathcal{K}$ depending on $\tau$ by the Koebe Lemma and a constant $K_{2}$ depending only on nonflatness such that for any $(c, z) \subset(c, y)$

$$
\left|f^{m}(z, y)\right| /|(z, y)|>\mathcal{K}(\tau) K_{2} D f^{m}\left(c_{1}\right)^{1 / l} .
$$

(2) If $\left|f^{m} A\right|>\tau\left|f^{m} B\right|$ for some $\tau>1$ then for some constant $K_{3}$ depending only on nonflatness

$$
\begin{gathered}
\left|f^{m-k}\left(f^{k} B\right)\right| /\left|f^{k} B\right|>\mathcal{C}(1) K_{3} D f^{m-k}\left(c_{1}\right)^{1 / l} \quad \text { and } \\
m-k>\nu(1 / \tau) \quad \text { where } \quad \nu(\varepsilon)=\min \left\{n:\left|c_{n}-c\right|<\varepsilon\right\} .
\end{gathered}
$$


Proof. (1) Suppose first that $\left|f^{m} A\right|<\tau\left|f^{m} B\right|$. We apply the Koebe Lemma (Lemma 2.1(3)) for $f^{m-1}$ on $f A$ and obtain

$$
\frac{\left|f^{m}(z, y)\right|}{|f(z, y)|}>\mathcal{C}\left(\frac{1}{\tau}\right) \frac{\left|f^{m}(c, z)\right|}{|f(c, z)|}, \quad \frac{\left|f^{m}(c, y)\right|}{|f(c, y)|}>\mathcal{C}\left(\frac{1}{\tau}\right) D f^{m-1}\left(c_{1}\right),
$$

and as $(c, y)=A$

$$
\frac{\left|f^{m}(z, y)\right|}{|f(z, y)|}>\mathcal{C}\left(\frac{1}{\tau}\right) \frac{\left|f^{m} A\right|}{|f A|}, \quad \frac{\left|f^{m} A\right|}{|f A|}>\mathcal{C}\left(\frac{1}{\tau}\right) D f^{m-1}\left(c_{1}\right) .
$$

If $\left|f^{m} A\right|>\tau\left|f^{m} B\right|$ but $\left|f^{m} A\right| /|A|<\kappa\left|f^{m} B\right| /|B|$ then in particular $B<\kappa A$ and by Lemma $2.2(3)$

$$
\frac{\left|f^{m} A\right|}{|f A|}=\frac{\left|f^{m} A\right|}{|A|} \frac{|A|}{|f A|}<\kappa \frac{\left|f^{m} B\right|}{|B|} \frac{|A|}{|f A|}=\frac{\left|f^{m} B\right|}{|f B|} \kappa \frac{|A|}{|f A|} \frac{|f B|}{|B|}<\frac{\left|f^{m} B\right|}{|f B|} .
$$

Therefore by Lemma 2.1(2)

$$
\begin{aligned}
\frac{\left|f^{m} A\right|}{|f A|}>D f^{m-1}\left(c_{1}\right), \\
\frac{\left|f^{m}(z, y)\right|}{|f(z, y)|}>\min \left\{\frac{\left|f^{m} A\right|}{|f A|}, \frac{\left|f^{m} B\right|}{|f B|}\right\}=\frac{\left|f^{m} A\right|}{|f A|} .
\end{aligned}
$$

In both cases of (1) we obtain by Lemma 3.1

$$
\left|f^{m} A\right| /|A|>\mathcal{C}(1 / \tau) K D f^{m}\left(c_{1}\right) .
$$

By nonflatness we have $|f(y, z)| /|(y, z)|>\mathcal{O}|f A| /|A|$, hence

$$
\frac{\left|f^{m}(z, y)\right|}{|(z, y)|}=\frac{\left|f^{m}(z, y)\right|}{|f(z, y)|} \frac{|f(z, y)|}{|(z, y)|}>\mathcal{C}\left(\frac{1}{\tau}\right) \frac{\left|f^{m} A\right|}{|f A|} \mathcal{O} \frac{|f A|}{|A|}=\mathcal{C}\left(\frac{1}{\tau}\right) \mathcal{O} \frac{\left|f^{m} A\right|}{|A|}
$$

This proves $(1)$ with $\mathcal{K}(\tau)=\mathcal{C}(1 / \tau)^{2}$.

In order to prove (2) we see that $\left|f^{m-k} \widetilde{\Delta}_{m-k}(c)\right|=\left|f^{m} B\right|<1 / \tau$, which implies the estimate on $m-k$. The other estimate follows again from the Koebe Lemma and Lemma 3.1.

Proposition 3.1. Let $\tau>1$. There are constants $\mathcal{M}$ (depending only on nonflatness) and $\mathcal{K}=\mathcal{K}(\tau)$ such that for any $n$ and any $(z, y) \subset(c, y)=$ $\widetilde{\Delta}_{n}(c)$ there is a sequence of nonnegative integers $\left(r_{j}\right)_{j \geq 0}$ such that

$$
\frac{\left|f^{n}(z, y)\right|}{|(z, y)|}>\mathcal{K}(\tau) D f^{r_{0}}\left(c_{1}\right)^{1 / l} \prod_{j \geq 1} \mathcal{M} D f^{r_{j}}\left(c_{1}\right)^{1 / l}
$$

$\sum_{j \geq 0} r_{j}=n$ and $r_{j}>\nu(1 / \tau)$ for $j \geq 1(\nu$ was defined in Lemma 3.2(2)).

Proof. Let $\kappa$ be as in Lemma 2.2(3). Let $(c, y) \subset(c, x)=\widetilde{\Delta}_{k}(c) \in \Delta_{n}$. If either

$$
\tau\left|f^{n}(y, x)\right|>\left|f^{n}(c, y)\right| \quad \text { or } \quad \kappa \frac{\left|f^{n}(y, x)\right|}{|(y, x)|}>\frac{\left|f^{n}(c, y)\right|}{|(c, y)|}
$$


then the proposition follows from Lemma 3.2(1) with $r_{0}=n$ and $r_{j}=0$ for $j \geq 1$. In particular, this proves the proposition for any $n<\nu(1 / \tau)$.

Set $\mathcal{O}=\mathcal{C}(1) K_{3}$ from Lemma $3.2(2)$ and $\mathcal{M}=\kappa \mathcal{O}$. We may assume that the proposition is true for any $k<n$ and we prove it by induction for $n$ when

$$
\tau\left|f^{n}(y, x)\right|<\left|f^{n}(c, y)\right| \text { and } \kappa \frac{\left|f^{n}(y, x)\right|}{|(y, x)|}<\frac{\left|f^{n}(c, y)\right|}{|(c, y)|} .
$$

By Lemma 2.1(2) we have

$$
\frac{\left|f^{n}(z, y)\right|}{|(z, y)|}>\min \left\{\frac{\left|f^{n}(c, y)\right|}{|(c, y)|}, \frac{\left|f^{n}(y, x)\right|}{|(y, x)|}\right\}>\kappa \frac{\left|f^{n}(y, x)\right|}{|(y, x)|} .
$$

On the other hand, we have

$$
\frac{\left|f^{n}(y, x)\right|}{|(y, x)|}=\frac{\left|f^{n-k}\left(f^{k}(y, x)\right)\right|}{\left|f^{k}(y, x)\right|} \frac{\left|f^{k}(y, x)\right|}{|(y, x)|}=\frac{\left|f^{n-k} \widetilde{\Delta}_{n-k}(c)\right|}{\left|\widetilde{\Delta}_{n-k}(c)\right|} \frac{\left|f^{k}(y, x)\right|}{|(y, x)|} .
$$

As

$$
\begin{aligned}
\tau\left|f^{n-k} \widetilde{\Delta}_{n-k}(c)\right| & =\tau\left|f^{n}(y, x)\right|<\left|f^{n}(c, y)\right| \\
& =\left|f^{n} \widetilde{\Delta}_{n}(c)\right|<\left|f^{n-k}\left(\Delta_{n-k}(c) \backslash \widetilde{\Delta}_{n-k}(c)\right)\right|<1
\end{aligned}
$$

we may apply Lemma 3.2(2) and estimate the first quotient by $\mathcal{O} D f^{n-k}\left(c_{1}\right)^{1 / l}$, and additionally obtain $n-k>\nu(1 / \tau)$.

Using the inductive hypothesis we estimate the second quotient by

$$
\mathcal{K}(\tau) D f^{r_{0}}\left(c_{1}\right)^{1 / l} \prod_{j \geq 1} \mathcal{M} D f^{r_{j}}\left(c_{1}\right)^{1 / l}
$$

(with $\sum_{j \geq 1} r_{j}=k-r_{0}$ and $\left.r_{j}>\nu(1 / \tau)\right)$ as $(y, x) \subset(c, x)=\widetilde{\Delta}_{k}(c)$. Therefore

$$
\begin{aligned}
\left|f^{n}(z, y)\right| /|(z, y)| & >\kappa\left|f^{n}(y, x)\right| /|(y, x)| \\
& >\kappa \mathcal{O} D f^{n-k}\left(c_{1}\right)^{1 / l} \mathcal{K}(\tau) D f^{r_{0}}\left(c_{1}\right)^{1 / l} \prod_{j \geq 1} \mathcal{M} D f^{r_{j}}\left(c_{1}\right)^{1 / l} \\
& =\mathcal{K}(\tau) D f^{r_{0}}\left(c_{1}\right)^{1 / l} \mathcal{M} D f^{n-k}\left(c_{1}\right)^{1 / l} \prod_{j \geq 1} \mathcal{M} D f^{r_{j}}\left(c_{1}\right)^{1 / l},
\end{aligned}
$$

which completes the proof as $n-k>\nu(1 / \tau)$.

COROLlary 3.1. If $f$ satisfies the summability condition on the critical trajectory then it does so on the central slopes. In other words,

$$
\sum D f^{n}\left(c_{1}\right)^{-1 / l}<\infty \quad \text { implies } \sum^{*}\left(\frac{\left|f^{n} \widetilde{\Delta}_{n}(c)\right|}{\left|\widetilde{\Delta}_{n}(c)\right|}\right)^{-1}<\infty .
$$


Proof. By Proposition 3.1 we can choose $\tau$ so large that

$$
d_{r}:=\left(\mathcal{M D} f^{r}\left(c_{1}\right)^{1 / l}\right)^{-1}<1
$$

for $r>\nu(1 / \tau)$. The conclusion follows from Lemma 3.3.

LEMMA 3.3. Let $\left(D_{n}\right)$ and $\left(d_{i}\right)$ be two sequences of positive numbers, with $d_{i}<1$ for all $i$ and $\sum d_{i}<\infty$. Assume that for any $n$ there is a sequence $\left(r_{j}\right)$ of nonnegative integers with $\sum_{j \geq 0} r_{j}=n$ such that

$$
D_{n}<\prod_{j} d_{r_{j}} .
$$

Then

$$
\sum D_{n}<\infty
$$

Proof. Take $N$ such that $\sum_{i>N} d_{i}<1$. Then

$$
\sum D_{n} \leq \sum_{j_{1}=1}^{\infty} d_{1}^{j_{1}} \sum_{j_{2}=1}^{\infty} d_{2}^{j_{2}} \ldots \sum_{j_{N}=1}^{\infty} d_{N}^{j_{N}} \sum_{k=0}^{\infty}\left(\sum_{n>N} d_{n}\right)^{k}<\infty .
$$

Proposition 3.2. If $f$ satisfies the Collet-Eckmann condition, i.e. $\liminf D f^{n}\left(c_{1}\right)^{1 / n}=\lambda_{C}>1$, then const $\lambda_{C}^{n / l}$ is an asymptotical estimate for

1) the central slopes: $\left|f^{n} \widetilde{\Delta}_{n}(c)\right| /\left|\widetilde{\Delta}_{n}(c)\right|$,

2) the central periodic orbits: $D f^{n}(p)$ with $f^{n}(p)=p \in \Delta_{n}(c)$,

3) the slopes on the c-level: $\left|f^{n} \widetilde{\Delta}_{n}^{i}\right| /\left|\widetilde{\Delta}_{n}^{i}\right|$,

4) the intervals of monotonicity on the c-level: $\left|\Delta_{n}^{i}\right|^{-1}, \Delta_{n}^{i} \in \Delta_{n} \backslash \Delta_{n+1}$,

5) any intervals of monotonicity: $\left|\Delta_{n}^{i}\right|^{-1}$,

$6)$ the derivatives at the preimages of the critical point: $D f^{n}(z)$ with $f^{n}(z)=c$,

7) the derivatives at any periodic point: $D f^{n}(p)$ with $f^{n}(p)=p$.

The estimate is asymptotical in the following sense. For any $\lambda<\lambda_{C}^{1 / l}=$ : $\lambda_{0}$ there is a constant $K(\lambda)$ such that any of the above quantities may be estimated from below by $K \lambda^{n}$. In the proof we use Proposition 3.1 for the central slopes and the relations between different hyperbolic structures established in [N1-N3].

Proof. Let $\lambda_{i}, i=1, \ldots, 7$, be the estimate of the corresponding term in the list, for example

$$
\lambda_{6}=\lim \inf \min _{z_{n}=c} D f^{n}(z)^{1 / n} .
$$

For any $\lambda<\lambda_{0}$ choose $\tau$ so that $\mathcal{M} D f^{r}\left(c_{1}\right)^{1 / l}>\lambda^{r}$ for $r>\nu(1 / \tau)$. This proves $\lambda_{1} \geq \lambda$ and hence also $\lambda_{1} \geq \lambda_{0}$. As $\left|f^{n} \widetilde{\Delta}(c)\right| /\left|\widetilde{\Delta}_{n}(c)\right| \rightarrow \infty$ one can 
estimate $\lambda_{2}$ with the Koebe Lemma by

$$
D f^{n}(p)>\mathcal{C}(1)\left|f^{n}(p, y)\right| /|(p, y)|>\text { const } \lambda^{n},
$$

which gives $\lambda_{2} \geq \lambda_{0}$. One can also estimate similarly to the proof of the Proposition 3.1 that $\lambda_{2} \geq \lambda_{1}$. For $\lambda_{3} \geq \lambda_{1}$ one uses the method described in $[\mathrm{N} 2, \mathrm{~N} 3]$ with $*(n)$ and $* *(n)$ intervals. In case of [N3] ( $f$ nonsymmetric) one has to revise carefully the proof and use the methods from the present paper. As the central intervals are part of the intervals on the $c$-level we obtain $\lambda_{3}=\lambda_{1}$. Next, $\lambda_{4} \geq \lambda_{3}$ is obvious as the intervals on the $c$-level are each a union of two intervals from $\widetilde{\Delta}_{n}$. Any interval of monotonicity $\Delta_{n}^{i}$ is an interval on the $c$-level for some $N \geq n$; this proves $\lambda_{5}=\lambda_{4}$

The derivatives at the preimages of the critical points can be estimated by the slopes on the intervals from $\widetilde{\Delta}_{n}$, which proves $\lambda_{6} \geq \lambda_{3}$. By [N1], $\lambda_{6}=\lambda_{7}$, and $\lambda_{7} \geq \lambda_{5}$ (it is not explicitly stated but one can deduce it from the methods used there). Obviously $\lambda_{2} \geq \lambda_{7}$. We have therefore

$$
\lambda_{0} \leq \lambda_{1} \leq \lambda_{3} \leq \lambda_{4}=\lambda_{5} \leq \lambda_{7}=\lambda_{6} \leq \lambda_{2} .
$$

The details are omitted.

Rem ark 3.1. The estimate by $\lambda_{C}^{1 / l}$ is sometimes the best available. For $f(x)=4 x(1-x)$ we have $\lambda_{C}=f^{\prime}(0)=4$ and $D f^{n}(p)^{1 / n}=2=4^{1 / \ell}$ (by diffeomorphic conjugation to a tent map with slope 2 ). The same (with some constant) holds for $\left|\Delta_{n}^{i}\right|^{-1}$ and therefore for any slope starting at the $c$-level.

Remark 3.2. The number $\lambda_{x}$ as defined in the Introduction is not smaller than $\lambda_{7}$. This also gives the estimate of the metric entropy of $\mu$.

4. The derivatives. In this section we estimate the derivative at an arbitrary point by some derivative on the critical trajectory, the distance of the image of the point to the closest critical value on its branch of monotonicity and some slope. The idea is to use this in the estimation of the Perron-Frobenius operator which is essentially the sum of the inverses of the derivatives. What one needs is the summability of the inverses of the corresponding slopes.

LEMMA 4.1. Let $(\alpha, \beta) \in \Delta_{n}, f^{k}(\alpha)=c, y \in(\alpha, \beta)$, and $\left|f^{n}(\alpha, y)\right| \leq$ $\left|f^{n}(y, \beta)\right|$. Then for some constant independent of $y,(\alpha, \beta)$ and $n$ we have

$$
D f^{n}(y) \geq \text { const } D f^{n-k-1}\left(c_{1}\right)^{1 / l}\left|y_{n}-c_{n-k}\right|^{1-1 / l} \frac{\left|y_{k}-\alpha_{k}\right|}{|y-\alpha|} .
$$

Pr o of. By the chain rule we have

$$
D f^{n}(y)=D f^{k}(y) D f\left(y_{k}\right) D f^{n-k-1}\left(y_{k+1}\right) \text {. }
$$


Obviously

$$
\begin{aligned}
D f\left(y_{k}\right) & =\frac{\left|D f\left(y_{k}\right)\right|}{\left|f\left(y_{k}\right)-c_{1}\right|^{1-1 / l}}\left|y_{k+1}-c_{1}\right|\left(\frac{\left|y_{n}-c_{n-k}\right|}{\left|y_{k+1}-c_{1}\right|}\right)^{1 / l}\left|y_{n}-c_{n-k}\right|^{-1 / l} \\
& >\mathcal{O C}(1) D f^{n-k-1}\left(c_{1}\right)^{1 / l}\left|y_{k+1}-c_{1}\right| \frac{1}{\left|y_{n}-c_{n-k}\right|^{1 / l}},
\end{aligned}
$$

by nonflatness and the Koebe Lemma for $f^{n-k-1}$ on $\left(y_{k+1}, c_{1}\right)$.

By Lemma 2.1(4) we have

$$
\begin{aligned}
D f^{k}(y) & >\frac{\left|y_{k}-\alpha_{k}\right|\left|\beta_{k}-y_{k}\right|}{\left|\beta_{k}-\alpha_{k}\right|} \frac{|\beta-\alpha|}{|y-\alpha||\beta-y|} \\
& =\frac{\left|y_{k}-\alpha_{k}\right|}{|y-\alpha|} \frac{\left|\beta_{k}-y_{k}\right|}{\left|\beta_{k}-\alpha_{k}\right|} \frac{|\beta-\alpha|}{|\beta-y|}>\frac{\left|y_{k}-\alpha_{k}\right|}{|y-\alpha|} \frac{\left|\left(\beta_{k}, y_{k}\right)\right|}{\left|\left(\beta_{k}, c\right)\right|} .
\end{aligned}
$$

Similarly, using the assumption $\left|\beta_{n}-y_{n}\right|>\left|y_{n}-\alpha_{n}\right|$ we have

$$
\begin{aligned}
D f^{n-k-1}\left(y_{k+1}\right) & >\frac{\left|y_{n}-\alpha_{n}\right|\left|\beta_{n}-y_{n}\right|}{\left|\beta_{n}-\alpha_{n}\right|} \frac{\left|\beta_{k+1}-\alpha_{k+1}\right|}{\left|y_{k+1}-\alpha_{k+1}\right|\left|\beta_{k+1}-y_{k+1}\right|} \\
& =\left|y_{n}-c_{n-k}\right| \frac{\left|\beta_{n}-y_{n}\right|}{\left|\beta_{n}-\alpha_{n}\right|} \frac{1}{\left|y_{k+1}-c_{1}\right|} \frac{\left|f\left(\beta_{k}, c\right)\right|}{\left|f\left(\beta_{k}, y_{k}\right)\right|} \\
& >\frac{1}{2}\left|y_{n}-c_{n-k}\right| \frac{1}{\left|y_{k+1}-c_{1}\right|} \frac{\left|f\left(\beta_{k}, c\right)\right|}{\left|f\left(\beta_{k}, y_{k}\right)\right|} .
\end{aligned}
$$

Therefore we use Lemma $2.2(2)$ on $\left(\beta_{k}, y_{k}\right) \subset\left(\beta_{k}, c\right)$ and get

$$
D f^{n}(y)>\text { const }\left|y_{n}-c_{n-k}\right|^{1-1 / l} D f^{n-k-1}\left(c_{1}\right)^{1 / l} \frac{\left|y_{k}-\alpha_{k}\right|}{|y-\alpha|} .
$$

R e mark 4.1. In this lemma we split any interval $(\alpha, \beta) \in \Delta_{n}$ into two subintervals. The image under $f^{n}$ of the splitting point $\gamma$ is exactly in the middle of the image of $f^{n}(\alpha, \beta)$. We estimate the derivative $D f^{n}(y)$ using $\alpha$ or $\beta$ depending on whether $y \in(\alpha, \gamma)$ or $y \in(\gamma, \beta)$.

This method is not dynamic because if $(\alpha, \beta) \in \Delta_{n} \cap \Delta_{n+1}$ (i.e. both $f^{n}$ and $f^{n+1}$ are monotone on $\left.(\alpha, \beta)\right)$ the points $\gamma$ corresponding to $f^{n}$ and $f^{n+1}$ will usually be different. A dynamic method of splitting $(\alpha, \beta)$ was used in $[\mathrm{KN}]$. Let $m>n$ be minimal such that $(\alpha, \beta) \notin \Delta_{m}$; then $(\alpha, \beta)=(\alpha, \gamma) \cup(\gamma, \beta)$ and both subintervals are in $\Delta_{m}$. This $\gamma$ defines a splitting as long as $(\alpha, \beta)$ is a maximal interval of monotonicity.

Proposition 4.1. If

$$
\sum_{m}^{*}\left(\left|f^{m} \widetilde{\Delta}_{m}(c)\right| /\left|\widetilde{\Delta}_{m}(c)\right|\right)^{-1}<\infty
$$


then there exists a constant $K$ such that for any $n$

$$
\sum_{\widetilde{\Delta}_{n}^{i} \in \widetilde{\Delta}_{n}}^{* *(n)}\left(\left|f^{n} \widetilde{\Delta}_{n}^{i}\right| /\left|\widetilde{\Delta}_{n}^{i}\right|\right)^{-1} \leq K .
$$

Proof. Let $N$ be such that

$$
\sum_{m>N}^{*}\left(\left|f^{m} \widetilde{\Delta}_{m}(c)\right| /\left|\widetilde{\Delta}_{m}(c)\right|\right)^{-1} \leq 1 / 4
$$

and $\delta$ such that if $\left|f^{m} \widetilde{\Delta}_{m}(c)\right|<\delta$ then $m>N$. Set $K=2 / \delta$. We prove the lemma by induction. Split the sum (**) into two parts depending on the length of $f^{n} \widetilde{\Delta}_{n}$ :

$$
\sum_{\widetilde{\Delta}_{n}^{i} \in \widetilde{\Delta}_{n}}^{* *(n)}\left(\frac{\left|f^{n} \widetilde{\Delta}_{n}^{i}\right|}{\left|\widetilde{\Delta}_{n}^{i}\right|}\right)^{-1}=\sum_{\left|f^{n} \widetilde{\Delta}_{n}^{i}\right| \geq \delta}^{* *(n)}\left(\frac{\left|f^{n} \widetilde{\Delta}_{n}^{i}\right|}{\left|\widetilde{\Delta}_{n}^{i}\right|}\right)^{-1}+\sum_{\left|f^{n} \widetilde{\Delta}_{n}^{i}\right|<\delta}^{* *(n)}\left(\frac{\left|f^{n} \widetilde{\Delta}_{n}^{i}\right|}{\left|\widetilde{\Delta}_{n}^{i}\right|}\right)^{-1} .
$$

If the length is greater than $\delta$ then the corresponding part of the sum is smaller than $\sum \widetilde{\Delta}_{n}^{i} / \delta<1 / \delta$. This proves the lemma for initial $n$ 's.

Observe that for any $\widetilde{\Delta}_{n}^{i} \in \widetilde{\Delta}_{n}$ there exists a $k<n$ such that $f^{k} \widetilde{\Delta}_{n}^{i}=$ $\widetilde{\Delta}_{n-k}(c)$. If we estimate the second part of the sum then

$$
\left|f^{n-k} \widetilde{\Delta}_{n-k}(c)\right|=\left|f^{n} \widetilde{\Delta}_{n}^{i}\right|<\delta
$$

and hence $n-k>N$. Moreover, the slope $\left|f^{k} \widetilde{\Delta}_{n}^{i}\right| /\left|\widetilde{\Delta}_{n}^{i}\right|$ may be estimated by the slope $\left|f^{k} \widetilde{\Delta}_{k}^{j}\right| /\left|\widetilde{\Delta}_{k}^{j}\right|$ of one of the two intervals from $\widetilde{\Delta}_{k}$ (Lemma 2.1(2)) which have a common endpoint with $\widetilde{\Delta}_{n}^{i}$. Obviously any such interval $\widetilde{\Delta}_{k}^{j}$ may be used at most twice.

The other part of the sum can hence be estimated by

$$
\begin{aligned}
\sum_{\left|f^{n} \widetilde{\Delta}_{n}^{i}\right|<\delta}^{* *(n)}\left(\frac{\left|f^{n} \widetilde{\Delta}_{n}^{i}\right|}{\left|\widetilde{\Delta}_{n}^{i}\right|}\right)^{-1} & =\sum_{\left|f^{n} \widetilde{\Delta}_{n}^{i}\right|<\delta}^{* *(n)}\left(\frac{\left|f^{n-k} f^{k} \widetilde{\Delta}_{n}^{i}\right|}{\left|f^{k} \widetilde{\Delta}_{n}^{i}\right|} \frac{\left|f^{k} \widetilde{\Delta}_{n}^{i}\right|}{\left|\widetilde{\Delta}_{n}^{i}\right|}\right)^{-1} \\
& <\sum_{n-k>N}^{*} 2 \sum_{\widetilde{\Delta}_{k}^{j} \in \widetilde{\Delta}_{k}}^{* *(k)}\left(\frac{\left|f^{n-k} \widetilde{\Delta}_{n-k}(c)\right|}{\left|\widetilde{\Delta}_{n-k}(c)\right|} \frac{\left|f^{k} \widetilde{\Delta}_{k}^{j}\right|}{\left|\widetilde{\Delta}_{k}^{j}\right|}\right)^{-1} \\
& =2 \sum_{n-k>N}^{*}\left(\frac{\left|f^{n-k} \widetilde{\Delta}_{n-k}(c)\right|}{\left|\widetilde{\Delta}_{n-k}(c)\right|}\right)^{-1} \sum_{\widetilde{\Delta}_{k}^{j} \in \widetilde{\Delta}_{k}}^{* *(k)}\left(\frac{\left|f^{k} \widetilde{\Delta}_{k}^{j}\right|}{\left|\widetilde{\Delta}_{k}^{j}\right|}\right)^{-1} \\
& <2 \cdot \frac{1}{4} \sum_{\widetilde{\Delta}_{k}^{j} \in \widetilde{\Delta}_{k}}^{* *(k)}\left(\frac{\left|f^{k} \widetilde{\Delta}_{k}^{j}\right|}{\left|\widetilde{\Delta}_{k}^{j}\right|}\right)^{-1},
\end{aligned}
$$


where the number 2 appears as we may select twice the "worse" of the two possible $\widetilde{\Delta}_{k}^{j}$. By previous considerations and induction we finish the proof with

$$
\sum_{\widetilde{\Delta}_{n}^{i} \in \widetilde{\Delta}_{n}}^{* *(n)}\left(\frac{\left|f^{n} \widetilde{\Delta}_{n}^{i}\right|}{\left|\widetilde{\Delta}_{n}^{i}\right|}\right)^{-1}<\frac{1}{\delta}+2 \cdot \frac{1}{4} K=\frac{K}{2}+\frac{K}{2}=K .
$$

COROLlary 4.1. Let $\left(K_{n}\right)$ be a sequence such that

$$
\sum_{\widetilde{\Delta}_{n}^{i} \in \widetilde{\Delta}_{n}}^{* *(n)}\left(\frac{\left|f^{n} \widetilde{\Delta}_{n}^{i}\right|}{\left|\widetilde{\Delta}_{n}^{i}\right|}\right)^{-1} \leq K_{n}
$$

Then for the iterates of the Perron-Frobenius operator $\mathcal{L}$ one has

$$
\mathcal{L}^{n}(1)(x) \leq 2 \sum_{r=0}^{n-1} K_{n-r} D f^{r}\left(c_{1}\right)^{-1 / l}\left|x-c_{r+1}\right|^{-(1-1 / l)} .
$$

Proof. Use Lemma 4.1. By definition $\mathcal{L}^{n}(1)(x)=\sum_{f^{n} y=x} D f^{n}(y)^{-1}$. The conclusion follows by comparing $\left|y_{k}-\alpha_{k}\right| /|y-\alpha|$ with the slope of $f^{k}$ on one of the two intervals from $\widetilde{\Delta}_{k}$ with endpoint $\alpha$ (and $\alpha_{k}=c$ ).

COROLlaRY 4.2. Under the summability condition on the critical trajectory (see Lemma 3.1) the same estimate for $\mathcal{L}^{n}$ holds with $K_{n}=K$.

Proof. Use Corollaries 3.1 and 4.1 .

Remark 4.2. One may also use [NS3] where it was proven that under the summability condition one has $\left|f^{-k}(c-\varepsilon, c+\varepsilon)\right| / \varepsilon<$ const with a uniform constant. This corresponds to $\sum|y-\alpha| /\left|y_{k}-c\right|<$ const, uniformly.

R e mark 4.3. Corollary 4.2 gives a natural (and independent of [NS3]) proof of the existence of an invariant density and the bounds on its singularities.

Proof. Consider $\left(\frac{1}{n} \sum_{k<n} \mathcal{L}^{k} 1\right)$ and use compactness.

Remark 4.4. In the situation of Corollary 4.1 one can also estimate the derivative of $\mathcal{L}^{n} 1$. Namely, since on any branch of monotonicity $(\alpha, \beta)$ one has by Lemma $2.1(5)$

$$
\left|\frac{D^{2} f^{n}(y)}{D f^{n}(y)^{2}}\right|<\frac{2}{\min \left\{\left|f^{n}(\alpha, y)\right|,\left|f^{n}(y, \beta)\right|\right\}},
$$

it follows that 


$$
\begin{aligned}
\left|\left(\mathcal{L}^{n} 1\right)^{\prime}(x)\right| & =\left|\sum_{f^{n} y=x} \frac{D^{2} f^{n}(y)}{D f^{n}(y)^{2}} \frac{1}{D f^{n}(y)}\right| \\
& <2 \sum_{r=0}^{n-1} K_{n-r} D f^{r}\left(c_{1}\right)^{-1 / l}\left|x-c_{r+1}\right|^{-(2-1 / l)}
\end{aligned}
$$

\section{References}

[BL] A. Blokh and M. Lyubich, Measurable dynamics of S-unimodal maps of the interval, Ann. Sci. École Norm. Sup. 24 (1991), 545-573.

[CE1] P. Collet and J.-P. Eckmann, Iterated Maps on the Interval as Dynamical Systems, Birkhäuser, Boston 1980.

[CE2] - - - Positive Liapounov exponents and absolute continuity for maps of the interval, Ergodic Theory Dynamical Systems 3 (1983), 13-46.

[K] G. Keller, Exponents, attractors and Hopf decomposition for interval maps, ibid. 10 (1990), 717-744.

[KN] G. Keller and T. Nowicki, Spectral theory, zeta functions and the distribution of periodic points for the Collet-Eckmann maps, Comm. Math. Phys. 149 (1992), $31-69$.

[L] F. Ledrappier, Some properties of absolutely continuous invariant measures on an interval, Ergodic Theory Dynamical Systems 1 (1981), 77-93.

[MS] W. de Melo and S. van Strien, One Dimensional Dynamic, book manuscript.

[M] M. Misiurewicz, Absolutely continuous invariant measures for certain maps of an interval, Publ. Math. I.H.E.S. 53 (1981), 17-51.

[N1] T. Nowicki, On some dynamical properties of S-unimodal maps of an interval, Fund. Math. 126 (1985), 27-43.

[N2] - Symmetric S-unimodal mappings and positive Liapunov exponents, Ergodic Theory Dynamical Systems 5 (1985), 611-616.

[N3] - A positive Liapunov exponent for the critical value of an S-unimodal mapping implies uniform hyperbolicity, ibid. 8 (1988), 425-435.

[NS1] T. Nowicki and S. van Strien, Hyperbolicity properties of multimodal ColletEckmann maps without Schwarzian derivative conditions, Trans. Amer. Math. Soc. 321 (1990), 793-810.

[NS2] - - - Absolutely continuous invariant measures for $C^{2}$ unimodal maps satisfying Collet-Eckmann conditions, Invent. Math. 93 (1988), 619-635.

[NS3] - , - Invariant measures exist under a summability condition for unimodal maps, ibid. 105 (1991), 123-136.

[Sz] B. Szewc, Perron-Frobenius operator in spaces of smooth functions on an interval, Ergodic Theory Dynamical Systems 4 (1984), 613-641.

[Y] L.-S. Young, Decay of correlations for certain quadratic maps, Comm. Math. Phys. 146 (1992), 123-138.

\section{INSTITUTE OF MATHEMATICS}

WARSAW UNIVERSITY

BANACHA 2

02-097 WARSZAWA, POLAND 